\title{
A Phase Field Method for Tomographic Reconstruction from Limited Data
}

\author{
Russell J. Hewett \\ rhewett@mit.edu
}

lan Jermyn

i.h.jermyn@durham.ac.uk

Michael T. Heath

heath@illinois.edu

Farzad Kamalabadi

farzadk@illinois.edu

\author{
Imaging and Computing Group \\ Department of Mathematics \\ Massachusetts Institute of Technology \\ Cambridge, MA
}
Department of Mathematical Sciences
Durham University
Durham, UK

Department of Computer Science

University of Illinois at

Urbana-Champaign

Urbana, IL

Department of Electrical and Computer

Engineering

University of Illinois at

Urbana-Champaign

Urbana, IL

\begin{abstract}
Classical tomographic reconstruction methods fail for problems in which there is extreme temporal and spatial sparsity in the measured data. Reconstruction of coronal mass ejections (CMEs), a space weather phenomenon with potential negative effects on the Earth, is one such problem. However, the topological complexity of CMEs renders recent limited data reconstruction methods inapplicable. We propose an energy function, based on a phase field level set framework, for the joint segmentation and tomographic reconstruction of CMEs from measurements acquired by coronagraphs, a type of solar telescope. Our phase field model deals easily with complex topologies, and is more robust than classical methods when the data are very sparse. We use a fast variational algorithm that combines the finite element method with a trust region variant of Newton's method to minimize the energy. We compare the results obtained with our model to classical regularized tomography for synthetic CME-like images.
\end{abstract}

\section{Motivation and Background}

Our knowledge of the physical processes that drive the sun is far from complete. Phenomena such as active regions, solar flares, coronal mass ejections (CMEs), and solar wind, all of which contribute to geoeffective events, collectively referred to as space weather, are 
not well understood [四, yet are of critical importance due to modern society's reliance on technologies that can be disrupted by these events.

Understanding such activity requires knowledge of physical parameters of the solar corona (or solar atmosphere), such as temperature and electron density [ $\mathbf{\theta}$ ? ], but such knowledge is hard to come by, particularly for local, short-lived events such as CMEs. One promising source of information is provided by satellite based solar telescopes called coronographs. Images from these instruments are formed by line-of-sight integrals of the free electron density in the corona. In principle, these images can be used to reconstruct the density field.

Coronagraph measurements of the sun are available from at most three perspectives at any given time, due to observatories along the Earth-Sun line and the dual STEREO spacecraft [Q], resulting in extremely limited observations of space weather events. Classical 3D tomographic reconstruction of the solar corona relies on a dense set of measured data from all around the sun and exploits solar rotation or dynamic modeling [] partially to resolve difficulties due to the limited observation configuration.

For direct CME imaging, however, the short lifetime of CMEs, long acquisition times, and inadequacies in current dynamical models render these methods unusable. In addition, while data are available for some CMEs from up to three observation points, it is more common to have observations of any given event from only two view points. As such, any CME tomography model must be robust to very small amounts of data. In practice, such reconstruction is a nontrivial problem. Mumford-Shah type models [ $\square]$ have previously been pro-

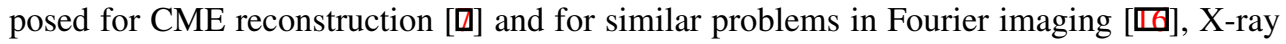
tomography [ㅁ] , and near-infrared spectroscopic imaging [ब]. Frazin et al. [0] represent the electron density by two distinct functions, corresponding to the CME and the background. These functions are Tikhonov-regularized with two different regularization parameters, allowing more variation in the CME region, while forcing the background to be smoother. However, solutions necessarily have a discontinuity on the boundary, while in reality there is only one continuous distribution of electron density. In addition, the use of distance function level sets to represent the segmentation means that heuristics are required to handle complex topologies, such as for solutions with multiple disjoint or embedded connected components, or regions with holes in them [ष] , situations that often arise in CMEs.

In this paper, we present a method for reconstruction that uses an auxiliary segmentation to constrain the density, but that resolves the above issues by using the phase field level set framework to represent the segmentation. We thereby eliminate important topological limitations present in previous work, and allow for smooth enforcement of two different regularization regimes, while retaining robustness to sparse data. Moreover, we compute maximum a priori (MAP) estimates using an efficient optimization algorithm that significantly reduces computational complexity compared to classical gradient descent.

The joint segmentation-tomography model is discussed in Sec. 2, while in Sec. 3 we describe the optimization algorithm. In Sec. 4 we describe and discuss test results on synthetic images and on a 2D slice of a 3D simulation of a CME, as used for testing in [Q].

\section{A Phase Field Model for Segmentation and Tomography}

We seek to infer the electron density $f: \Omega \rightarrow \mathbb{R}$ in a local section of the solar corona $\Omega$ and a segmentation classifying a subset $R \subset \Omega$ as part of a CME, given a set of coronagraphs $Y$ and prior knowledge $K$, e.g. parameter values. We represent the segmentation by a phase field function, $\phi: \Omega \rightarrow \mathbb{R}$, and construct a probability distribution $P(f, \phi \mid Y, K)$ representing 
our knowledge of $f$ and $\phi$, from which the MAP estimate, $(\hat{f}, \hat{\phi})=\arg \max _{(f, \phi)} P(f, \phi \mid Y, K)$, can be determined. Using $P(Y \mid f, K)=P(Y \mid f, \phi, K)$, applying Bayes' theorem, and writing everything in terms of energy functionals (negative log probabilities), yields the equivalent minimization problem:

$$
(\hat{f}, \hat{\phi})=\underset{(f, \phi)}{\arg \min } E(Y \mid f, K)+E(f \mid \phi, K)+E(\phi \mid K) .
$$

\subsection{Tomographic Imaging Model}

The data $Y$ are a set of measurements $\left\{y_{j}\right\}_{j \in[1 . . m]}$, with $y_{j}: \Gamma \rightarrow \mathbb{R}$, where $\Gamma$ is the measurement domain and $y_{j}=h_{j}(f)+n_{j}$, where $h_{j}$ is a tomographic projection operator (and thus $y_{j}$ is a projection or line-of-sight measurement) and $n_{j} \sim \mathcal{N}\left(0, \sigma^{2}\right)$. Consequently, the energy due to measured data is

$$
E(Y \mid f, K)=\sum_{j} \frac{1}{2 \sigma^{2}} \int_{\Gamma}\left(y_{j}-h_{j}(f)\right)^{2}
$$

\subsection{Segmentation Model}

We use the phase field framework to construct the segmentation prior energy $E(\phi \mid K)$ of (1). A phase field is a level set function, $\phi: \Omega \rightarrow \mathbb{R}$, representing the region $R=\{x \in \Omega \mid \phi(x)>0\}$. As prior energy for the segmentation, we use the Ginzburg-Landau functional []],

$$
E(\phi \mid K)=\int_{\Omega}\left\{c_{1} \frac{1}{2} \nabla \phi \cdot \nabla \phi+c_{2}\left(\frac{1}{4} \phi^{4}-\frac{1}{2} \phi^{2}\right)+c_{3}\left(\phi-\frac{1}{3} \phi^{3}\right)\right\},
$$

where $c_{1}, c_{2}$, and $c_{3}$ are free parameters. The last two terms are a double well potential where, for $\left|c_{3}\right|<c_{2}$, local minima occur at $\phi= \pm 1$, with a local maximum at $\phi=\frac{c_{3}}{c_{2}}$. Coupled with the smoothing effect of the first term, the potential terms ensure that, away from the region boundary and for fixed $R, \phi$ takes the values 1 in $R$ and -1 in $\Omega \backslash R$. Near the boundary, there is a smooth transition from -1 to 1 across a diffuse interface zone of width roughly $4 \sqrt{c_{1} / c_{2}}$. The effective energy controlling $R$ is then a linear combination of the length (surface area) of the boundary and the area (volume) of the interior of $R[\square]$ for 2D (3D) regions.

Because $\phi$ is not constrained a priori to take on a particular form (e.g. a distance function), ad hoc methods for adding connected components or holes in an existing domain are unnecessary: region components can be produced and eliminated in a natural way []].

\subsection{Electron Density Model}

We define the energy $E(f \mid \phi, K)$, which couples the phase field to the density, by

$$
E(f \mid \phi, K)=\int_{\Omega}\left\{\frac{1}{2}\left(\lambda_{+} \phi_{+}+\lambda_{-} \phi_{-}\right)(\nabla f \cdot \nabla f)-c_{4} \nabla \phi \cdot \nabla f\right\},
$$

where $\phi_{ \pm}=(1 \pm \phi) / 2$ act as pseudo-indicator functions for the CME and background regions. The first term defines distinct Tikhonov regularization parameters, $\lambda_{ \pm}$, for the interior and exterior of $R$. The second term favours large inward pointing $\nabla f$ on the boundary, because CMEs generally have sharply higher densities than the background. Thus, like [ $\square]$, we model the background as smoother than the CME, and with a very different density, but 
unlike [ $\square]$, the diffuse nature of the phase field interface allows the regularization parameter to change smoothly, though rapidly, across the interface. We therefore only need one density function.

\section{Variational Energy Minimization}

To compute the MAP estimate, we thus minimize the joint segmentation-tomography energy functional

$$
\begin{array}{r}
E_{\mathrm{JST}}(f, \phi)=\sum_{j} \frac{1}{2 \sigma^{2}} \int_{\Gamma}\left(y_{j}-h_{j}(f)\right)^{2}+\int_{\Omega}\left\{\frac{1}{2}\left(\lambda_{+} \phi_{+}+\lambda_{-} \phi_{-}\right)(\nabla f \cdot \nabla f)-c_{4} \nabla \phi \cdot \nabla f\right. \\
\left.+c_{1} \frac{1}{2} \nabla \phi \cdot \nabla \phi+c_{2}\left(\frac{1}{4} \phi^{4}-\frac{1}{2} \phi^{2}\right)+c_{3}\left(\phi-\frac{1}{3} \phi^{3}\right)\right\} .
\end{array}
$$

In line with previous applications, we initially attempted to optimize $E_{\mathrm{JST}}$ using a splitstep gradient descent method, alternately finding zeros of the components of $\nabla E_{\mathrm{JST}}$, i.e. the functional derivatives in each variable. Traditionally, the subproblems are solved by introducing a fictitious time dependence and solving the resulting PDE using an explicit finite difference method. However, the small time step required for stability leads to slow convergence and implicit methods that allow larger time steps are impractical due to increased computational complexity from the nonlinearity in the phase field potential. Additionally, the split-step method complicates the implementation: it is necessary to solve each subproblem only partially, at least early in the descent, to avoid getting stuck in local minima, but determining how far to go before switching subproblems is nontrivial.

To resolve these issues, we minimize $E_{\text {JST }}$ in both $f$ and $\phi$ simultaneously using a finite element discretization and a trust-region-based variation on Newton's method, the Levenberg-Marquardt method [ $[\boldsymbol{G}, \mathbb{\square}]$. In this approach, the length of the descent step is dependent upon the minimization algorithm and the local shape of the objective function, and is not explicitly constrained by the discretization.

Let $u=(f, \phi) \in V$ and $E=E_{\mathrm{JST}}: V \rightarrow \mathbb{R}$. We seek the $u_{*} \in V$ that minimizes $E$. Rather than directly computing $F(u)$ and $D F(u)$, the functional gradient and Hessian operator of $E$, and applying the standard Newton's method for optimization $[\mathbf{Q}, \mathbf{Q}, \mathbf{\square}]$ in the finite difference framework, we apply Newton's method in the weak sense. The weak form of the EulerLagrange equations $F(u)$ is found by determining the first directional derivative of $E$,

$$
\langle F(u), v\rangle=\left.\frac{\mathrm{d}}{\mathrm{d} \tau} E(u+\tau v)\right|_{\tau=0},
$$

where $\langle a, b\rangle$ is the $L^{2}$ inner product on $\Omega$. The Hessian, given by the linearized bilinear form of (6), is

$$
\langle D F(u)[w], v\rangle=\left.\frac{\mathrm{d}}{\mathrm{d} \tau}\langle F(u+\tau w), v\rangle\right|_{\tau=0} .
$$

For a fixed $u$, the Newton step is then the $w \in V$ for which

$$
\langle D F(u)[w], v\rangle=-\langle F(u), v\rangle \forall v \in V .
$$

The next Newton iterate is then $u+\alpha w$, where $\alpha \in(0,1]$ is a damping parameter. 


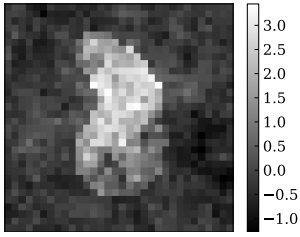

(a)

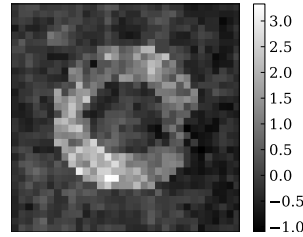

(b)

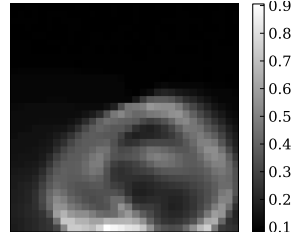

(c)

Figure 1: Test images. (a) 'kidney' and (b) 'annulus' images sampled from approximate model distribution. (c) 2D slice of 3D CME simulation. (Image source: [0])

When (8) is discretized using the finite element method and the step is applied iteratively, a linear system $\mathbf{A}_{k} \mathbf{w}_{k}=\mathbf{b}_{k}$ is obtained. At each step $k$ of the iteration, $\mathbf{A}_{k}$ and $\mathbf{b}_{k}$ are recomputed based on the current discretized solution $\mathbf{u}_{k}$. Solving for $\mathbf{w}_{k}$ at each iteration, the discrete Newton iteration $\mathbf{u}_{k+1}=\mathbf{u}_{k}+\alpha \mathbf{w}_{k}$, is repeated until the norm of the gradient $\mathbf{b}_{k}$ is sufficiently small. However, due to the nonconvexity of $E_{\mathrm{JST}}, \mathbf{A}_{k}$ is frequently not positive definite, particularly for early iterations, so $\mathbf{u}_{k}$ is not guaranteed to be a descent step. We apply the Levenberg-Marquardt method to guarantee positive definiteness. In this method, the update is the solution to the linear system $\left(\mathbf{A}_{k}+\mu \mathbf{I}\right) \mathbf{w}_{k}=\mathbf{b}_{k}$ where $\mu$ is a shift parameter chosen to make the system positive definite. We compare the results with the above method ('LM'), to the method of steepest descent ('SD'), obtained in a similar manner by solving for the $w$ for which $\langle I[w], v\rangle=-\langle F(u), v\rangle \forall v \in V$, where $I$ is the identity operator. For the method of steepest descent, a line search is used to compute a safe, optimal step length.

\section{Numerical Experiments}

We use two synthetic images and one approximation of a CME image (Fig. 1) to demonstrate the effectiveness of the phase field joint segmentation-tomography model. The 'kidney' shape in Fig. 1(a) and the 'annulus' in Fig. 1(b) were chosen because the kidney shape is relatively simple, but has a concave section, and the annulus is again simple but has a hole in the middle, which can cause issues with other segmentation procedures. These regions were represented by phase fields, and the electron density $f$ was then generated by sampling from the Gaussian probability distribution associated with the energy $E(f \mid \phi, K)$ with parameter values $c_{4}=1, \lambda_{+}=1$, and $\lambda_{-}=6$. The CME sample image Fig. 1(c) was generously provided to us by the authors of [D]. This image is a $2 \mathrm{D}$ slice from a $3 \mathrm{D}$ simulation of an October 28, 1998 CME [س]].

We approximate $f$ and $\phi$ by piecewise linear basis functions on a mesh formed by triangulating an equispaced Cartesian grid. Synthetic data were generated by applying a projection operator $\mathbf{H}$, a matrix whose rows are the discretized projection operators $h_{j}$, to the sample images. Noise was then added to these synthetic measurements, with $\sigma=1$. For data generation, $\mathbf{H}$ was computed using a high-order quadrature rule, whereas for reconstruction H was generated using a low-order quadrature rule to avoid "inverse crimes". We employed the parameter values that were used in generating the data for the two synthetic examples. For the CME image, the parameter values were determined heuristically to be $c_{1}=1, c_{4}=5$, $\lambda_{+}=50$, and $\lambda_{-}=300$. The values of $c_{2}$ and $c_{3}$ are given below for each reconstruction. For all three examples, the phase field was initialized to the neutral initialization $\phi=c_{3} / c_{2}$, 


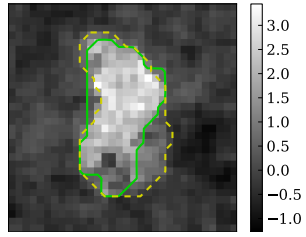

(a)

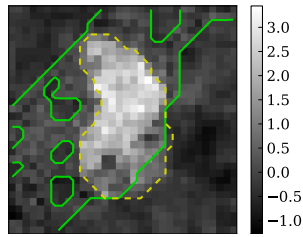

(b)

Figure 2: Segmentation-reconstructions: LM (left) and SD (right). Computed segmentations in solid green; true segmentations in dashed yellow.

\begin{tabular}{|c|c|c|c|c|c|c|c|c|}
\hline \multicolumn{2}{|c|}{ Experiment } & Size & $c_{1}$ & $c_{2}$ & $c_{3}$ & Iter. & Run Time & $E_{\mathrm{JST}}\left(u_{*}\right)$ \\
\hline \multirow{2}{*}{ Kidney } & $\mathrm{LM}$ & $32 \times 32$ & 1.0 & 110.0 & 17.0 & $\begin{array}{c}146 \\
5789\end{array}$ & $\begin{array}{c}55.1 \mathrm{~s} \\
130.2 \mathrm{~s}\end{array}$ & $\begin{array}{c}-31636.2118 \\
-23919.560\end{array}$ \\
\hline
\end{tabular}

Table 1: Parameter values and run time statistics for the kidney image, Fig. 1(a), using the LM and SD algorithms, for 32 equispaced observation angles and 32 projections per angle. Corresponding reconstructions are shown in Fig. 2.

i.e. the maximum of the phase field potential, while the initial density was $f=0$.

\subsection{Synthetic Images}

The first experiment compares the LM and SD algorithms. Fig. 2 shows segmentationreconstructions of the $32 \times 32$ kidney image using LM (left) and SD (right). The data consisted of 32 projections at each of 32 equispaced observation angles. The solid green line is the computed segmentation, while the dashed yellow line is the true segmentation. Parameter values and run time statistics are given in Tbl. 1. The result from SD is very poor compared with that from LM, and is typical of results we found with SD. The method often is caught in local minima representing low quality solutions, and the execution time is long. Due to this unreliability, we consider only the LM algorithm in the remainder of this paper.

The next experiment tests the method on increasingly sparse data. Fig. 3 shows results obtained on the annulus image, Fig. 1(b), with 32, 4, and 2, equispaced observation angles. The first column is the reconstruction; the second column is the phase field; the third column shows the computed (in solid green) and true (in dashed yellow) segmentations superimposed on the reconstruction. Tbl. 2 shows parameter values and run time statistics. We note that although the results necessarily decrease in quality as the number of observation angles decreases, in the two-angle case, a very limited data case, the essential structure of the annulus is still preserved. The gap in the annulus in the four-angle case can also be closed, by reducing $c_{3}$, but at the expense of additional spurious flanges like those in the bottom left corner. These flanges in both results are caused by the tendency of the smoothing term to smear high density regions along the observation direction. This is constrained by the segmentation energy, which is why the flanges are limited in extent, but in the absence of more data or prior knowledge, there is no way to eliminate them completely as possibilities.

The third experiment compares our model to Tikhonov regularization, i.e. $\phi \equiv 1$ or $\phi \equiv$ -1 . The results are shown in Fig. 4. The reconstruction in Fig. 4(b) is overly smooth in the annulus region, while the background is too rough in Fig. 4(c). In the four-angle 


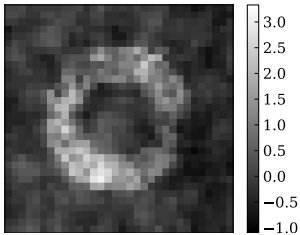

(a)

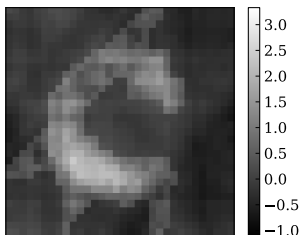

(d)

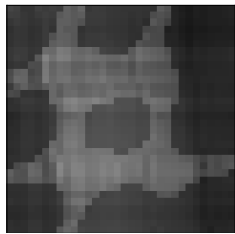

(g)

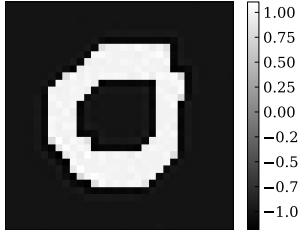

(b)

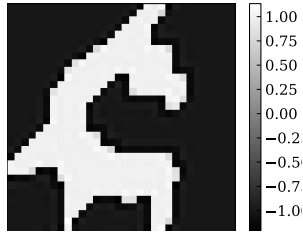

(e)

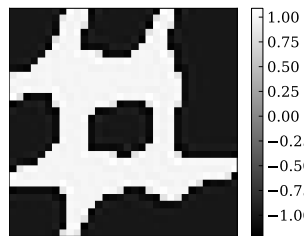

(h)

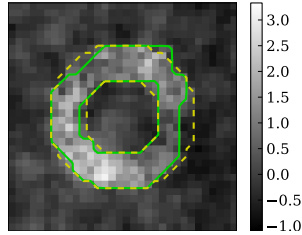

(c)

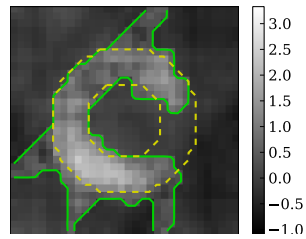

(f)

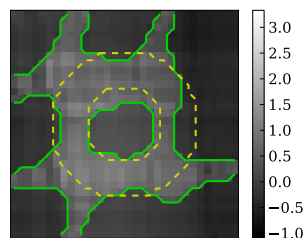

(i)

Figure 3: Reconstructions for annulus image. (a) reconstruction, (b) segmentation, (c) segmentation superimposed on reconstruction, for 32 equispaced observation angles with 32 projections per angle. (d), (e), and (f) same, for 4 equispaced observation angles. (g), (h), and (i) same, for 2 equally spaced observation angles. Computed segmentations in solid green; true segmentations in dashed yellow.

case, the ring shape is much easier to distinguish from the background in Fig. 4(d), and is a better reconstruction within the segmented region. For the two-angle case, the segmentationreconstruction method still computes something that is topologically an annulus, whereas the annulus is barely identifiable in the Tikhonov regularized results.

\subsection{Synthetic CME}

In this section, we present results from experiments on the CME image in Fig. 1(c). These are similar to the annulus experiments, except that we reconstruct the CME image from two angles, at $\pm 45^{\circ}$, and three angles, at $\pm 60^{\circ}$ and $0^{\circ}$, representing the best-case observing configurations for the current generation of satellites. The results are shown in Fig. 5, which is organized like Fig. 3. Tbl. 2 shows parameter values and run time statistics. Although the results are naturally not as good as those on the synthetic images, in all three cases there is a cavity within the CME, something that [ [] does not achieve. There is spurious region formation outside of the CME region, which might be an artifact of parameter settings that a fully automatic method would correct, or it might require a more complex model $E(f \mid \phi, K)$.

Finally, we compare our results to those obtained using Tikhonov regularization. The 


\begin{tabular}{|c|c|c|c|c|c|}
\hline Observation Angles & $c_{1}$ & $c_{2}$ & $c_{3}$ & Iter. & Run Time \\
\hline 32 & 1.0 & 140.0 & 21.9 & 247 & $82.0 \mathrm{~s}$ \\
4 & 1.0 & 225.0 & 33.0 & 67 & $10.5 \mathrm{~s}$ \\
2 & 1.0 & 225.0 & 30.0 & 25 & $3.14 \mathrm{~s}$ \\
\hline
\end{tabular}

Table 2: Parameter values and run time statistics for annulus image, Fig. 1(b). Corresponding reconstructions are shown in Fig. 3.

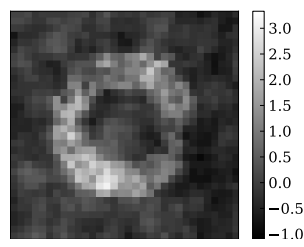

(a)

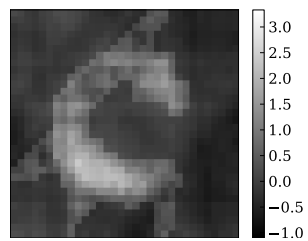

(d)

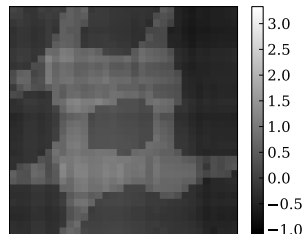

(g)

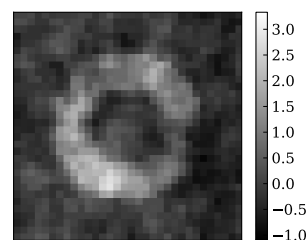

(b)

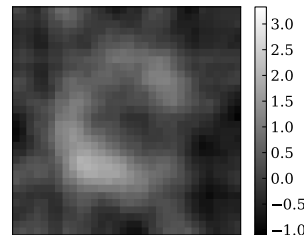

(e)

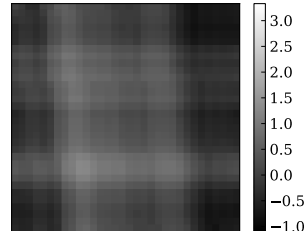

(h)

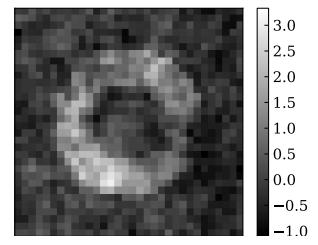

(c)

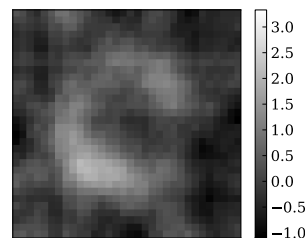

(f)

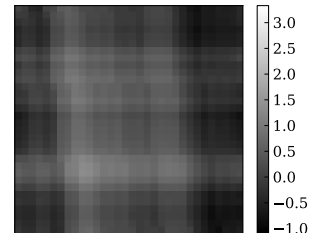

(i)

Figure 4: Comparison of joint segmentation-reconstruction with Tikhonov regularized reconstructions for annulus image. (a) Joint segmentation-reconstruction, (b) Tikhonov regularized reconstruction with $\lambda=\lambda_{-}$, (c) Tikhonov regularized reconstruction with $\lambda=\lambda_{+}$, for 32 equispaced observation angles and 32 projections per angle. (d), (e), and (f) same, for 4 equispaced observation angles. (g), (h), and (i) same, for 2 equispaced observation angles.

results are shown in Fig. 6. We see again that, even for the limited angle reconstructions, the joint segmentation-tomographic reconstructions have definition in the CME region that is not present in either of the Tikhonov regularized reconstructions.

\section{Conclusion}

We have presented a method for the reconstruction of CME electron density from coronagraphs taken at a small number of observation angles, using an auxiliary segmentation to constrain the density. Experiments show that our proposed method is significantly more 


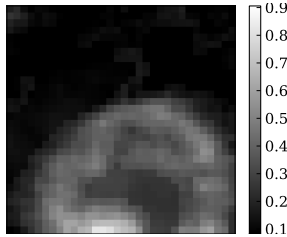

(a)

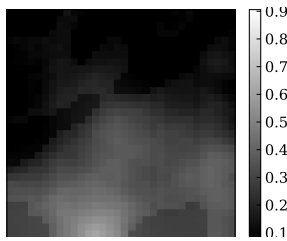

(d)

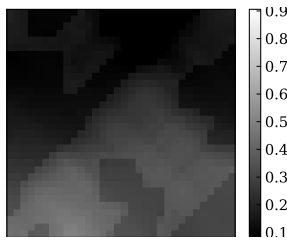

(g)

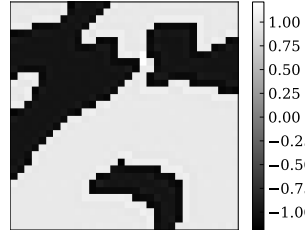

(b)

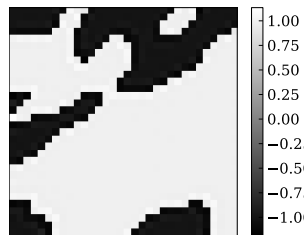

(e)

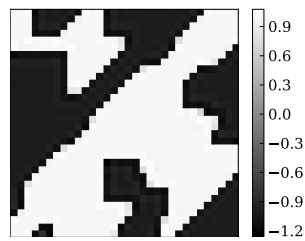

(h)

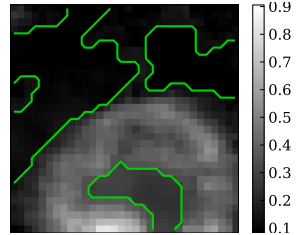

(c)

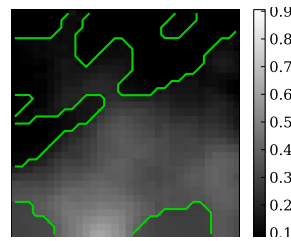

(f)

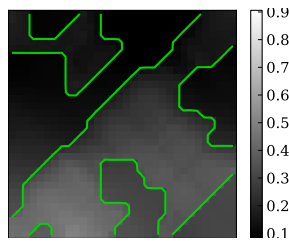

(i)

Figure 5: Reconstructions for CME image. (a) reconstruction, (b) segmentation, and (c) segmentation superimposed on reconstruction, for 32 equispaced observation angles with 32 projections per angle. (d), (e), and (f) same, for 3 equispaced observation angles. (g), (h), and (i) same, for 2 equispaced observation angles.

effective than Tikhonov regularized tomography alone, and resolves issues with CME topology and continuity of the density that affected previous work. Our model and optimization method easily extend for the full 3D CME reconstruction problem, though further work on parameter estimation is necessary to render the method automatic.

\section{References}

[1] M. J. Aschwanden. Physics of the Solar Corona. Springer-Verlag, Berlin, Germany, 2004.

[2] K.J. Bathe and A.P. Cimento. Some practical procedures for the solution of nonlinear finite element equations. Computer Methods in Applied Mechanics and Engineering, 22(1):59-85, 1980.

[3] M. D. Butala, R. J. Hewett, F. Kamalabadi, and R. A. Frazin. Dynamic three dimensional tomography of the solar corona. Solar Physics, 262:495-509, 2010.

[4] O. Cohen, I. V. Sokolov, I. I. Roussev, C. N. Arge, W. B. Manchester, T. I. Gombosi, R. A. Frazin, H. Park, M. D. Butala, F. Kamalabadi, and M. Velli. A semiempirical 


\begin{tabular}{|c|c|c|c|c|c|c|c|c|c|}
\hline Obs. Angles & $c_{1}$ & $c_{2}$ & $c_{3}$ & $c_{4}$ & $\lambda_{+}$ & $\lambda_{-}$ & $\sigma$ & Iter. & Run Time \\
\hline 32 & 1.0 & 200.0 & 10.0 & 5.0 & 50.0 & 300.0 & 1.0 & 27 & $10.2 \mathrm{~s}$ \\
4 & 1.0 & 150.0 & 10.0 & 5.0 & 50.0 & 300.0 & 1.0 & 27 & $4.0 \mathrm{~s}$ \\
2 & 1.0 & 150.0 & 20.0 & 5.0 & 50.0 & 300.0 & 1.0 & 79 & $11.2 \mathrm{~s}$ \\
\hline
\end{tabular}

Table 3: Parameter values and run time statistics for the CME image, Fig. 1(c). Corresponding reconstructions are shown in Fig. 3.

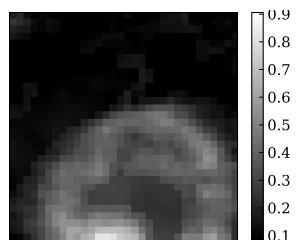

(a)

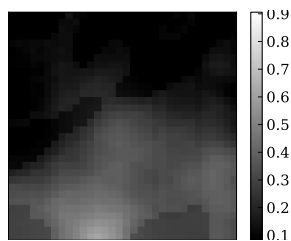

(d)

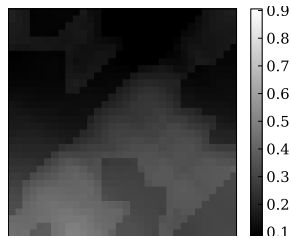

(g)

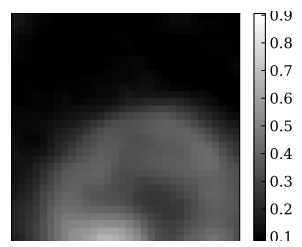

(b)

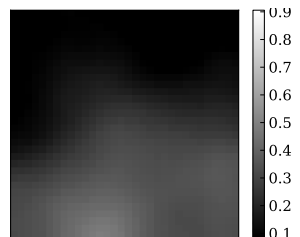

(e)

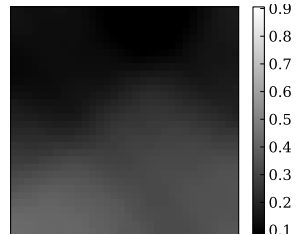

(h)

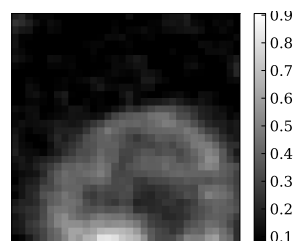

(c)

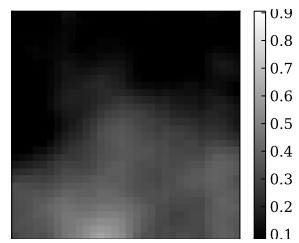

(f)

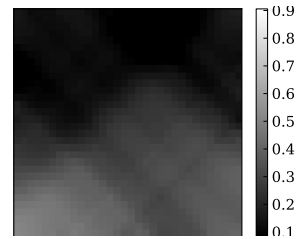

(i)

Figure 6: Comparison of joint segmentation-reconstruction with Tikhonov regularized reconstructions for CME image. (a) Joint segmentation-reconstruction, (b) Tikhonov regularized reconstruction with $\lambda=\lambda_{-}$, and (c) Tikhonov regularized reconstruction with $\lambda=\lambda_{+}$ for 32 equispaced observation angles and 32 projections per angle. (d), (e), and (f) same, for 3 equispaced observation angles. (g), (h), and (i) same, for 2 equispaced observation angles.

magnetohydrodynamical model of the solar wind. The Astrophysical Journal Letters, 654:L163-L166, 2007.

[5] A. Driesman, S. Hynes, and G. Cancro. The STEREO observatory. Space Science Reviews, 136(1):17-44, 2008.

[6] R. Fletcher. Practical Methods of Optimization. John Wiley \& Sons, Chichester, 2nd edition, 1987.

[7] R. A. Frazin, M. Jacob, W. B. Manchester, H. Morgan, and M. B. Wakin. Toward 
reconstruction of coronal mass ejection density from only three points of view. The Astrophysical Journal, 695:636-641, April 2009.

[8] M. Jacob, Y. Bresler, V. Toronov, X. Zhang, and A. Webb. Level-set algorithm for the reconstruction of functional activation in near-infrared spectroscopic imaging. Journal of Biomedical Optics, 11, 2006.

[9] K. Levenberg. A method for the solution of certain nonlinear problems in least squares. Quarterly of Applied Mathematics, 2(2):164-168, 1944.

[10] W.B. Manchester IV, A. Vourlidas, G. Tóth, N. Lugaz, I.I. Roussev, I.V. Sokolov, T.I. Gombosi, D.L. De Zeeuw, and M. Opher. Three-dimensional MHD simulation of the 2003 October 28 coronal mass ejection: Comparison with LASCO coronagraph observations. The Astrophysical Journal, 684:1448, 2008.

[11] D.W. Marquardt. An algorithm for least-squares estimation of nonlinear parameters. Journal of the Society for Industrial and Applied Mathematics, 11(2):431-441, 1963.

[12] H. Matthies and G. Strang. The solution of nonlinear finite element equations. International Journal for Numerical Methods in Engineering, 14(11):1613-1626, 1979.

[13] D. Mumford and J. Shah. Optimal approximations by piecewise smooth functions and associated variational problems. Communications on Pure and Applied Mathematics, 42(5):577-685, 1989.

[14] R. Ramlau and W. Ring. A Mumford-Shah level-set approach for the inversion and segmentation of X-ray tomography data. Journal of Computational Physics, 221(2): 539-557, 2007.

[15] M. Rochery, I. Jermyn, and J. Zerubia. Phase field models and higher-order active contours. In ICCV '05: Proceedings of the Tenth IEEE International Conference on Computer Vision, pages 970-976, 2005.

[16] J.C. Ye, Y. Bresler, and P. Moulin. A self-referencing level-set method for image reconstruction from sparse Fourier samples. International Journal of Computer Vision, 50(3):253-270, 2002. 